\title{
Molecular Characterization of GmFOX2, an Evolutionarily Highly Conserved Gene from the Mycorrhizal Fungus Glomus mosseae, Down-Regulated During Interaction with Rhizobacteria
}

\author{
Natalia Requena, Petra Füller, and Philipp Franken \\ Max-Planck-Institut für terrestrische Mikrobiologie, Karl-von-Frisch Strasse, 35043 Marburg, Germany \\ Accepted 2 July 1999.
}

\begin{abstract}
Arbuscular mycorrhizal (AM) fungi form the most widespread symbiosis of the plant kingdom. More than $80 \%$ of vascular plants are susceptible to colonization by the $\mathrm{zy}$ gomycetous fungi from the order Glomales, and profit significantly by the nutrient exchange between plant and fungus. However, knowledge of the biology of these fungi still remains elusive because of their obligate biotrophism and, up to now, unculturability. The molecular mechanisms underlying the pre-symbiotic stages and the cell-tocell communication between AM fungi and other soil microorganisms are, particularly, unknown. Here, we study these aspects by means of a molecular approach to monitor changes in the gene expression of the fungus Glomus mosseae (BEG12) in response to the rhizobacterium $\mathrm{Ba}$ cillus subtilis NR1. The bacterium was found to induce specific increases in mycelial growth as well as changes in expression of $\mathrm{GmFOX} 2$, a highly conserved gene encoding a multifunctional protein of the peroxisomal $\beta$-oxidation. We determined the gene structure and studied its expression in response to rhizobacteria at two time points. The results show that the fungus is able to change its gene expression in response to stimuli other than the plant.
\end{abstract}

Sustainable agriculture and ecosystem conservation have challenged scientists to consider the management of arbuscular mycorrhizal (AM) fungi as biofertilizers and/or bioprotectors as an alternative to standard fertilizers and pesticides (Barea and Jeffries 1995; Requena et al. 1996). The biotechnological applications of the symbiosis are, however, severely hindered because of the obligate biotrophism of the fungal partner (Mosse 1988). AM fungi germinate from asexual spores and form a limited, pre-symbiotic mycelium at the expense of their internal spore reserve in the absence of the host plant. However, they can only complete their life cycle after colonization of a host root (Bonfante and Bianciotto 1995).

Corresponding author: Natalia Requena; Telephone: 496421178 310; Fax: 496421178 309; E-mail: requena@mailer.uni-marburg.de

Nucleotide and/or amino acid sequence data can be found at the EMBL data base as accession number AJ243538.
Attempts to cultivate AM fungi in vitro led to the discovery that certain soil microorganisms can significantly increase the saprotrophic growth of the mycelia during pre-symbiosis (Hepper 1979). At this stage, competition of AM fungi with the rest of soil microbiota for nutrients is probably irrelevant since their saprotrophic growth is very limited and basically supported by the spore reserve. The positive influence of certain microorganisms during germination was early reported by Mosse (1959), who showed how the presence of some bacteria in the media accelerated the germination of AM fungal spores. Mayo et al. (1986) described how the addition of antibiotics to prevent bacterial contamination of the spores inhibited the germination of Glomus versiforme. Some of the bacteria involved in this effect were Pseudomonas and Corynebacterium spp. Many other authors later described similar effects (Azcón-Aguilar et al. 1986; Mugnier and Mosse 1987; Tylka et al. 1991; Carpenter-Boggs et al. 1995). Soil fungi have also been shown to exert a beneficial effect on AM fungal spore germination (Calvet et al. 1992). Besides the stimulatory effect on germination, the major role of most microorganisms interacting with AM fungi is a stimulatory effect on hyphal growth, branching pattern, and vegetative spore or auxiliary cell formation (Azcón-Aguilar et al. 1986; Mayo et al. 1986; Azcón 1989). These effects are species specific since not all bacterial or fungal treatments can induce them, and the magnitude of the effects is also dependent on the specific interaction (Azcón 1989; Tylka et al. 1991). There are also descriptions of some detrimental effects of soil microorganisms on AM fungi. Two AM fungi, Glomus etunicatum and $G$. mosseae, were able to germinate only after soil disinfection, showing the fungistatic ability of some soil microbes. Even more, fungistasis was restored when the original, nonsterile soil sievings were added to the pasteurized soil (Wilson et al. 1989).

Despite the extensive research done to elucidate the mechanisms responsible for the stimulatory effect of soil microbes on AM fungi (reviewed by Azcón-Aguilar and Barea 1992), it is still unknown how microbes might signal the fungus and elicit an increase in mycelial development (Requena 1998). We provide insights into the molecular events underlying the pre-symbiotic stages of the AM life cycle during the interac- 
tion between AM fungi and soil bacteria. Several bacteria originally isolated as PGPR (plant growth promoting rhizobacteria) (Requena et al. 1997) were screened for their ability to increase the hyphal development of G. mosseae (BEG 12) during pre-symbiosis. Sporocarps of G. mosseae (BEG 12), containing an average of 10 to 15 spores each, were purchased from Biorize (Dijon, France), and disinfected according to Budi et al. (in press). One hundred fifty sporocarps per treatment were germinated and grown in water-agar plates $(0.8 \%$ Bacto agar) buffered with morpholineethanesulfonic acid (MES; $10 \mathrm{mM}$ ) to $\mathrm{pH}$ 6.8. Six sporocarps per plate covered with a sterile cellophane membrane were inoculated with $10^{3} \mathrm{CFU}$ of the corresponding bacteria (Bacillus subtilis NR1, Mesorhizobium mediterraneum NR2, or PGPR A2) resuspended in $5 \mu \mathrm{l}$ of sterile saline solution $(\mathrm{NaCl} 0.85 \%)$. Control sporocarps received $5 \mu$ l of saline solution. The plates were Parafilm-sealed and placed upside-down in an incubator at $25^{\circ} \mathrm{C}$. Sporocarps were germinated and grown in the dark for 12 days, after which total hyphal length per sporocarp was recorded with a stereo-microscope and a 2.5 $\mathrm{mm}$ grid. The average germination time was 6 to 7 days for all treatments. The experiment was done three times and the results were subjected to analysis of variance followed by Duncan test for $P<0.05$. Among the screened bacteria, only $B$. subtilis NR1 was able to specifically increase by threefold the hyphal growth of G. mosseae (BEG 12) (Table 1). None of the bacteria had any significant effect on spore germination. However, when B. subtilis NR1 was inoculated on spores of another mycorrhizal fungus, Gigaspora rosea (BEG 9), the bacterium completely inhibited their germination. The effect was fungistatic because washing the spores and inoculation in fresh water agar medium restored their germination ability.

To gain insights into the molecular mechanisms underlying the interaction between $B$. subtilis NR1 and G. mosseae, differential RNA display was carried out on early fungal transcripts from sporocarps inoculated and not inoculated with $B$. subtilis NR1, following the inoculation procedure described above, at 1, 3, and 5 days after inoculation. RNA from 200 sporocarps per treatment was isolated (RNA easy; Qiagen, Hilden, Germany). Ten nanograms of total RNA was used for the differential display RT-PCR (reverse transcriptionpolymerase chain reaction) following the protocol of MartinLaurent et al. (1997). Differentially displayed fragments were gel isolated, re-amplified, and cloned in the TA vector from Invitrogen (Groningen, The Netherlands). A cDNA fragment of $375 \mathrm{bp}$ differentially displayed was isolated and sequenced. The deduced amino acid sequence from the cloned fragment showed high homology to the FOX2 protein from Neurospora crassa and to the human homologue $17 \beta$-hydroxysteroid dehydrogenase (HDS) type IV (Fossa et al. 1995; Carstensen et al. 1996). The expression of the gene was studied by means of quantitative RT-PCR with an internal competitive standard, on $50 \mathrm{ng}$ of total RNA from the set of sporocarps harvested $24 \mathrm{~h}$ after inoculation. RNA calibration was done by Northern (RNA) blot with a probe containing the 28S and 18S rRNA genes from $G$. mosseae (Franken and Gianinazzi-Pearson 1996). cDNA was synthesized with an oligo (dT) primer. PCR was performed with specific primers for a GmFOX2 fragment spanning an intron to avoid possible genomic DNA contaminations. Primer sequences were as follows: sense primer (P0),
5'-CAG GCC CTT CAA CAA GTT CTT TG-3' and antisense (P5), 5'-GCA TCA TCA CAT TGA AAT CGC C-3'. The drawing in Figure 1 illustrates the procedure for the quantitative RT-PCR with a competitive mimic construct (Clontech Technologies, Palo Alto, CA). Arrows indicate primer positions. Portions $(5 \%)$ of the cDNA were amplified with these primers in the presence of fivefold serial dilutions of the mimic starting with 50 amol (lanes 1 to 4). PCR was performed in the presence of $50 \mu \mathrm{Ci}$ labeled $\alpha^{32} \mathrm{P}$-dATP. Following 30 cycles of PCR amplification, $40 \%$ of the PCR product was resolved in a $4 \%$ acrylamide gel and exposed for $30 \mathrm{~min}$ to an X-ray film. Arrow heads indicate the approximate dilution at which the sample and the mimic had similar concentrations. In the treatment control the intensity of the bands corresponding to GmFOX2 and to the mimic construct is similar between dilutions 2 and 3, while in the case of sporocarps inoculated with $B$. subtilis NR1, the intensity of the bands is similar between dilutions 3 and 4. Therefore, it can be deduced that the RNA for $G m F O X 2$ was fivefold lower in samples inoculated with the bacterium. Densitometric analysis is shown in Table 2.

The cDNA fragment obtained from the differential RNA display was used as a probe to screen a genomic library of $G$. mosseae (Hosny et al. 1999). The fragment was PCR labeled with $50 \mu \mathrm{Ci} \alpha^{32} \mathrm{P}$-dATP, using specific primers. The primer sense sequence was 5'-CAG GCC CTT CAA CAA GTT CTT TG-3' and the antisense sequence was 5'-CCA GAA GTA GGG ATG GGT TTC-3'. Twenty-five clones, all containing the cDNA fragment, were isolated. One clone containing an $11-\mathrm{kb} E c o$ RI insert was further digested with $\mathrm{XbaI}$ and the fragments PCR-screened with the same specific primers. The positive fragments were subcloned and sequenced. On the basis of the sequence of the full genomic clone, specific primers were designed upstream and downstream of the putative ATG and TAA start and stop codons, respectively. RT-PCR on total RNA was performed with these primers to obtain the cDNA sequence. Analysis of the cDNA revealed that the gene contains eight introns, as already predicted from the similarity of the genomic clone of G. mosseae to the gene of $N$. crassa and its homologue in humans (Fig. 2). The introns are distributed at random throughout the entire gene and are typical fungal introns with a size between 69 and 198 bp (Gurr et al. 1987). They share, in most cases, a GT 5' sequence and a TAG in the $3^{\prime}$ end, with two exceptions, in the third (CAG) and the seventh (AAG) intron. The open reading frame (ORF) revealed a 1,015 amino acid protein with a calculated molecular mass of $120 \mathrm{kDa}$, organized as a multidomain structure with 67 and $64 \%$ homology to FOX2 from $N$. crassa and to $17 \beta-$ HDS IV from humans, respectively (Fig. 3). The G. mosseae gene was named $G m F O X 2$. The protein sequence contains a terminal SKL motif characteristic of proteins targeted to peroxisomes.

Table 1. Effect of the different bacterial treatments on Glomus mosseae (BEG 12) sporocarp germination and hyphal development

\begin{tabular}{lcc}
\hline Bacterial treatment & $\begin{array}{c}\text { Germination } \\
(\boldsymbol{\%})\end{array}$ & $\begin{array}{c}\text { Hyphal } \\
\text { growth }(\mathbf{m m})\end{array}$ \\
\hline Control & 70 & 42 \\
Bacillus subtilis NR1 & 72 & $150^{\mathrm{a}}$ \\
Mesorhizobium mediterraneum NR2 & 65 & 30 \\
PGPR A2 & 68 & 62 \\
\hline
\end{tabular}

a Significantly different according to Duncan's test for $P<0.05$. 


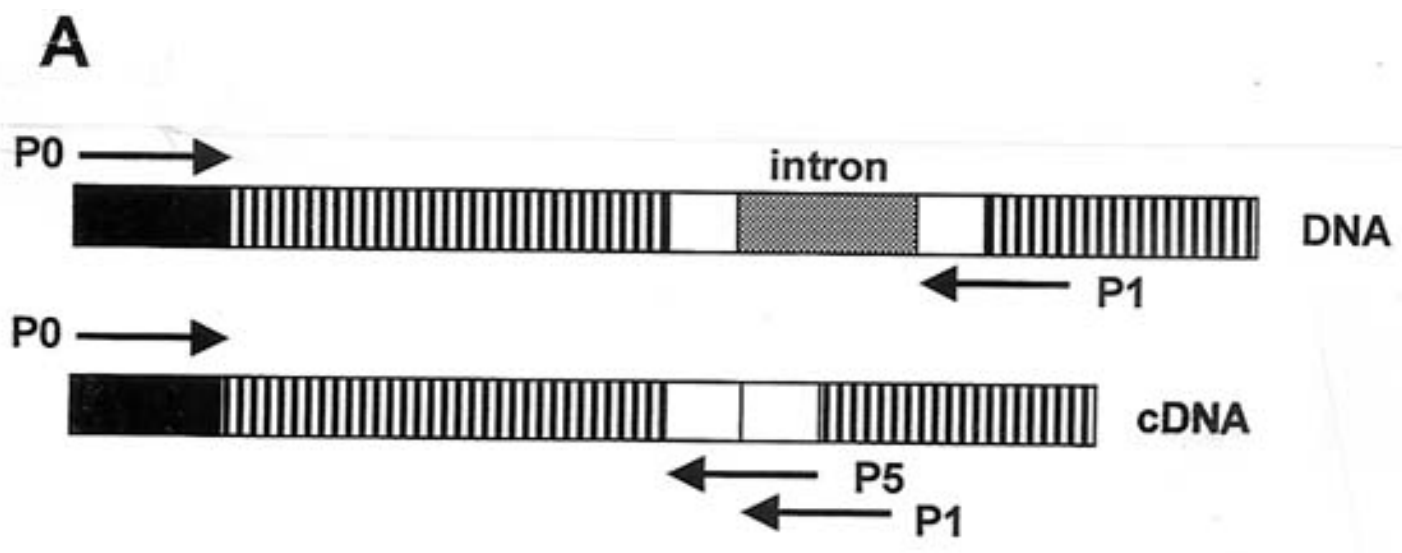

C1 B1

DNA

cDNA
C1 B1

\section{P0/P1}

C1 B1

444bp

$374 \mathrm{bp}$

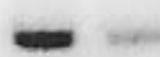

326bp cDNA

\section{P0/P5}

B
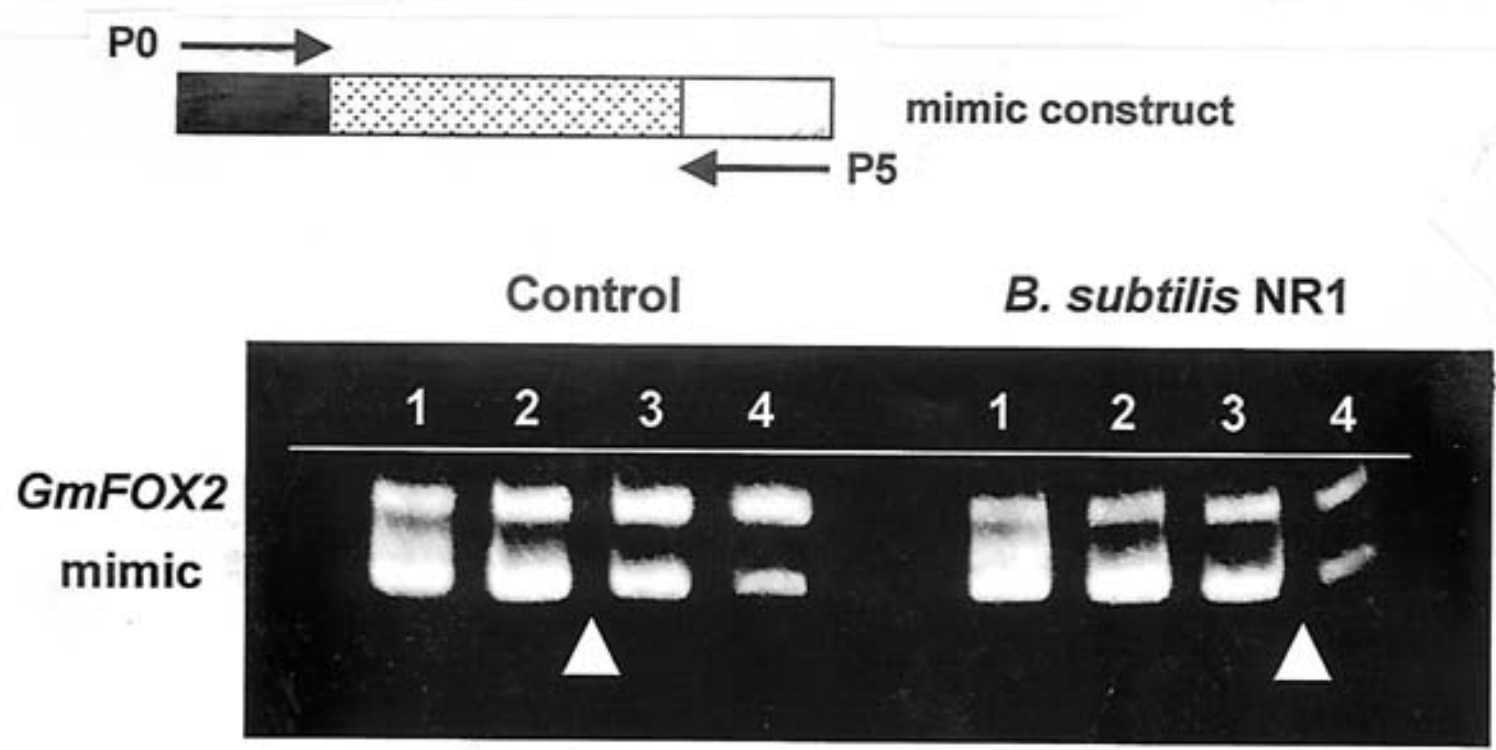

Fig. 1. Competitive reverse transcription-polymerase chain reaction (RT-PCR) analysis of GmFOX2 expressed in either control sporocarps or sporocarps inoculated with Bacillus subtilis NR1 after $24 \mathrm{~h}$ of inoculation. A, Design of different sets of primers annealing in the GmFOX2 gene and the RT-PCR products obtained. Only the combination of primers P0 and P5 (the latter spanning a 70-bp intron and therefore only binding to cDNA) successfully gave a single band. B, Structure of the mimic DNA used for quantification of changes in gene expression. The mimic construct possesses flanking region s identical to binding sites of primers P0 and P5, and a size to similar to that of the cDNA of GmFOX2. Serial fivefold dilutions (lanes 1 to 4 ) from 50 to 0.4 amol of the mimic DNA were added to each PCR tube, both to control and to inoculated treatments. Arrow heads indicate approximate dilution at which the sample and the mimic have similar concentrations. 
The amino acid alignment showed that GmFOX2 contains, as does its counterpart in humans, three different domains, only two of which are present in N. crassa (Fig. 4). The first domain in the N-terminal region is duplicated and it has homology to the NAD(P)-dependent dehydrogenase family that catalyzes the oxidation of hydroxyl groups of sugars, alcohols, aromatic compounds, steroids, and 3-hydroxyacyl esters (Persson et al. 1991). The enzymatic oxidation of D-3hydroxyacyl CoA during $\beta$-oxidation in peroxisomes has been attributed to this domain (Fossa et al. 1995; Leenders et al. 1996). The $\beta$-oxidation in peroxisomes uses different substrates than the corresponding pathway in mitochondria, and some molecules such as long-chain fatty acids, 2-methyl branched-chain fatty acids, or di- and trihydroxycholestanoic acids are specifically degraded in peroxisomes. Interestingly, the first domain was also characterized as the isozyme type IV of $17 \beta$-hydroxysteroid dehydrogenases, which catalyzes unidirectionally the oxidative inactivation of estradiol to estrone in mammals (Leenders et al. 1996; Carstensen et al. 1996). This domain seems to be cleaved from the rest of the protein when it has to control the level of active estrogens in the cell (Leenders et al. 1996). It is noteworthy that this first domain also shares a considerable homology (23\%) to NodG from Rhizobium meliloti, which is supposed to be involved in one of the enzymatic steps of the modification of lipo-chitooligosaccharide signals triggering the formation of the symbiosis with the plant (Sheldon et al. 1990). The central domain of GmFOX2 harbors the 2-enoyl-CoA hydratase that performs the second step of the $\beta$-oxidation in peroxisomes. The last domain is a sterol carrier-like protein 2, whose mutation causes impaired catabolism of methyl-branched fatty-acylCoAs in mammals (Seedorf et al. 1998). This domain is absent in the $N$. crassa FOX2 and also in the FOX2 protein from yeast. The role of this last domain could be to facilitate the transfer of different lipid molecules between membranes, as is the case in vitro (Leenders et al. 1996).

The expression of GmFOX2 was further studied by quantitative RT-PCR at two different time points of the growing period in the presence/absence of B. subtilis NR1 to gain insights into the gene regulation during the interaction with the bacterium. As an additional control a third set of sporocarps was inoculated with another bacterium, Mesorhizobium mediterraneum NR2, that did not produce any significant promotion of hyphal growth of G. mosseae. G. mosseae sporocarps (400 per treatment) were grown on water-agar plates after inoculation with either a saline solution or a bacterial suspension of $B$. subtilis NR1 or M. mediterraneum NR2 as described above. Half of the sporocarps of each treatment were harvested after $24 \mathrm{~h}$ and the rest after $120 \mathrm{~h}$. Total RNA was extracted from each treatment as described above and cDNA was synthesized with $50 \mathrm{ng}$ of total RNA, using an

Table 2. Densitometric analysis of the quantitative reverse transcriptionpolymerase chain reaction shown in Figure $1^{\mathrm{a}}$

\begin{tabular}{|c|c|c|c|c|c|c|c|c|}
\hline \multirow{2}{*}{$\begin{array}{l}\text { Polymerase } \\
\text { chain reaction } \\
\text { product }\end{array}$} & \multicolumn{4}{|c|}{ Control } & \multicolumn{4}{|c|}{ Bacillus subtilis NR1 } \\
\hline & 1 & 2 & 3 & 4 & 1 & 2 & 3 & 4 \\
\hline GmFOX2 & 37 & 44 & 48 & 44 & 28 & 26 & 25 & 14 \\
\hline Mimic & 80 & 74 & 45 & 23 & 86 & 72 & 47 & 13 \\
\hline
\end{tabular}

${ }^{\text {a }}$ Standard deviation for all data is $<0.008$. oligo (dT) primer. Competitive PCR with the mimic standard as control was performed with $5 \%$ of total cDNA in the presence of $50 \mu \mathrm{Ci}$ labeled $\alpha^{32} \mathrm{P}$-dATP with the same primers designed for the quantitative RT-PCR (P0 and P5). Three twofold mimic dilutions ranging from 10 to 2.5 amol were used. Radioactive products were resolved in a $4 \%$ acrylamide gel and exposed for $30 \mathrm{~min}$ to an X-ray film. RNA calibration was done by Northern blot with a probe containing the $28 \mathrm{~S}$ and $18 \mathrm{~S}$ rRNA genes. Figure 5 shows the results with the mimic dilution corresponding to 5 amol. Densitometric analysis is shown in Table 3. As demonstrated in the previous experiment, the expression of GmFOX2 in fungi growing in the presence of B. subtilis NR1 was down-regulated after $24 \mathrm{~h}$ in about fivefold, while inoculation with $M$. mediterraneum NR2 also produced a twofold down-regulation. After $120 \mathrm{~h}$, however, the expression levels in sporocarps inoculated with $B$. subtilis NR1 were similar to those from the control albeit still slightly smaller. $M$. mediterraneum NR2, however, produced a threefold down-regulation after $120 \mathrm{~h}$ (Fig. 5).

In this paper we report that the inoculation of the AM fungus $G$. mosseae with several rhizobacteria is able to promote changes in the early gene expression of this fungus. In particular the expression of GmFOX2 was found to be transiently down-regulated by fivefold after $24 \mathrm{~h}$ of inoculation with the bacterium $B$. subtilis NR1, which in addition promotes later (after 12 days) a remarkable increase in the pre-symbiotic development of the fungal growth. The molecular characterization of the gene has shown that it encodes a protein highly conserved within other organisms such as $N$. crassa or humans, showing, however, interesting and unique features with regards to its domain distribution in the mycorrhizal fungus. GmFOX2 resembles more the protein of $N$. crassa with respect to the dehydrogenase repeated motif, but, like its counterpart in humans, it has an extra domain at the end of the protein responsible for a different enzymatic activity that is missing in the $N$. crassa FOX2. AM fungi are among the oldest fungi on earth: they have been in existence for an estimated 400 million years (Berbee and Taylor 1992; Simon et al. 1993). We could speculate that, on the basis of the domain structures, the different homologues of GmFOX2 could have derived from a common ancestor closer to the mycorrhizal partner. Subsequent losses of some of the domains or rearrangements into different enzymes could have later originated the homologous proteins.

Lipids are the main carbon reserve of AM spores. During germination and further growth prior to the formation of the symbiosis, the fungus actively mobilizes its triacylglyceride reserve with a qualitative change in lipid composition and a negative balance of carbon in the total lipids after 16 days of growth. This indicates not only a re-allocation of carbon among lipids but also their use as a carbon source (Gaspar et al. 1994). The putative enzyme encoded by GmFOX2 is responsible for at least three different functions during the catabolism of long-chain fatty acids, suggesting an important role in fungal metabolism during pre-symbiosis. GmFOX2 homologues have been found to be mainly regulated at the transcriptional level (Kunau and Hartig 1992; Carstensen et al. 1996). While in mammals the expression of genes related to peroxisomal metabolism seems to be under the control of the PPARs (peroxisome proliferator activated receptors), in fungi the gene is known to be subjected to glucose repression. Glu- 


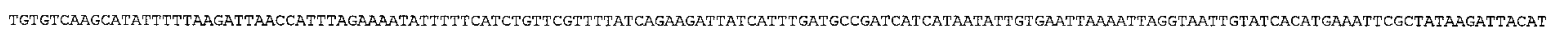
CGAAAAAAATCGAAAAAAAAATTTTCAGCCCTTTIT GAAGCTAATTCGCTTCGCTTGAAT GTTTTCGTCGAGTAAGTTTATATCATTTATATATATAAAACATTCGTTTAATTATTCAACATTGTTAAGTAAATAATAATAACTTTTAT ATATTAAGATTAACGTTGAAACGTTGTGITGAGTATTTTAATT TATAGGTTGGTTATAAATCAAAAATTTTAAT GTAGAATTTTCGCTTTAAGATACATGTTT GATCTAATTCGTTACAAGTTACAAATGCATGTTATGTTCAAAA.GTG TTAATTGCGAATTTAATTTCGCTATTCAGGTTAAATTCAAAGAAGGCACGAAAGCTAGGAATAAATCACAGCCATTCCAATATTITAAAAAACATTATTATATATAAATATCCTTTGTGAATAACT GAATILTGFCTCAAGACTTCGT

TTCGATGGACGCACTGT TATTGTTACTGGAGCTGGTGGT GTAA I TATAAAATTAAACT CATACTAGTTATT GAAGTGGCGCAGTTTTAGATTAACGCGTAATTTACTET TTCCATAAAGGTCTTGGACGTGCATATGCT TIAGCGTTC F $D$ G

GCGGCCGTGGCGTARTGTTGTAGTTAACGACCTTGGCGCGTCTCGAGGCGGGGATGGTTCATCATCTGCTTTTGCAGATAAAG AAFT GATGAAATTATCAAAGCTGGTGGCAAAGCAGPTTCGAATTATAATTCGGTT GAGGATGGT D E I I K A G G K A V S N Y N $S$ V E D

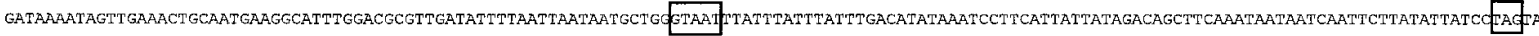
D K I V E T A M Intron 2

TTTTACGTGATAAGTCATTTGCAAGAATAACTGATTCAGATTGGGATCTTATTCAAGCTGTGCACGTCCGTGGTTCGTATAAAGT CACAAAGGCGGCTTGGGATATTTTCCGAAAACAAAAATTTGGCAGAATTATTAACACAGCATCAG

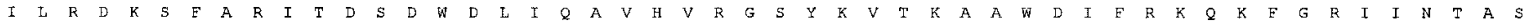

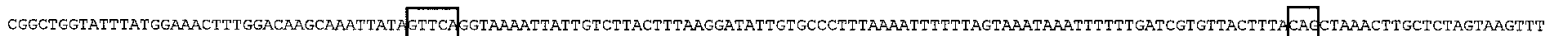

$\begin{array}{llllllllllllllllllllll}A & A & G & I & Y & G & N & F & G & Q & A & N & Y & \text { Intron } 3\end{array}$ T E T I A $\mathrm{K}$ E TCTATCTCAT GAATCTACTCAAGAAAATGGTAGTTTATTT GAAGTTGGAGCCGGTTTCGTTGCCAAGCTAAGATGGGAACGTTCTAARGGTGCAGTTTTCAAGGCCGATGATACTTTCTTACCTGGTACTGTCGCCGTAAAATGGAACGA

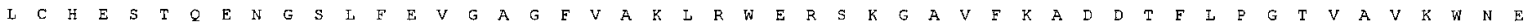
AATTACAGAGTTTACAAATCCTACTTATCCTACTTCACCGGGTGACGCCGATTTTGTTGGATTACTTGAGAAGGCAAAAGCGTTGCCTTCTAATCCAAAATCCGAAGACCTTAAATTCGATGGAAAAGTTGCTATTGTCACAGGAGCTGG

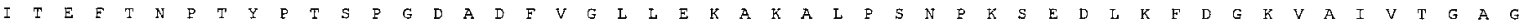
TGGTGGATTAGGTCGTGCATACGCTTTACTATTAGGAAAATTAGGGGCTTCAGTTGTTGTAAAT GACCTAGGAGTAAGTGCTCATGGTCAGGGAGCTACTTCAAGIGCAGCTGATAAAGTTGTTGAAGAAATTCGACAAGCTGGTGGTAA

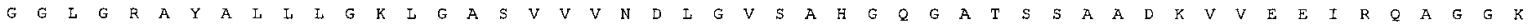
AGCTGTCGCCAATTATGATTCCGTAGAAGATGGTGAAAAAATTGTTGAACCGCTATTAAAGCTTTTGGTCGTGTTGATATTATTATCAAT AATGCAGGTATCCTGGAGAGATAAATCTTPTTGCTAGAATGACAGATCAGGACTGGGATTT

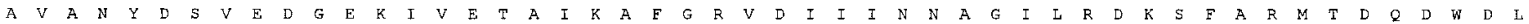
GGTTCAAAGGGTCCATTTGCGTGGAACTTACAAAGTIACTAAAGCTGCATGGCCTTATCTTACAAAACAAAAATATGGTCGTATAATCAACACAGCCTCTTCTGT CGGTTTATACGGCAACTTTGGTCAAGCAAATTACAGCACAGGTTA

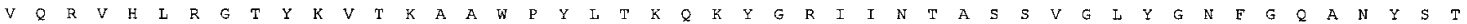

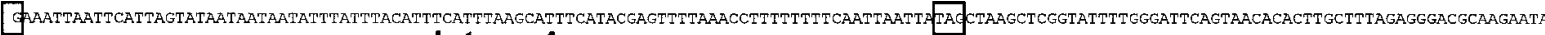

\section{Intron 4}

ATATTTTTGTCAACACAATTGCACCAAATGCCGGTACTCGAATGACAGCTACGATATGGCCACCGGATATGGTTGAGGCTTTCAAGCCTGATTATGTTGCACCATTTETATCATTCCTCGCTCATGAAGCATGTCCCTCGACTGGAAAT

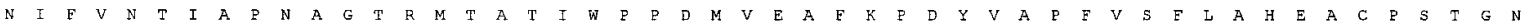
TTTT GAAGTGGGTGGT GGTTGGTTTGCTCAAGTTCGCTGGCAACGT GCTGGTGGAATTGGTTTTCCTACATCCAAAGCTCTAACTCCAGAAGATATAGCTTCAAAAATTGATATAATAACTAATTTT GACGATGGACGTGCCACCCATC

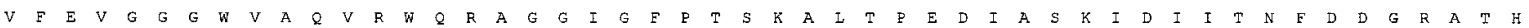
CAACAACTACACAACAGGCCCTTCAACAATTCTTTGAAAACT'T GCCAATGCGCAAAAATCTGAATCTGGACAATCTAAATCTAAAAGTAATAATAGTAAGATTGATGTGGARGCTGCAAAGAAACGAAAAATTCGAACCTCATGTCTTT

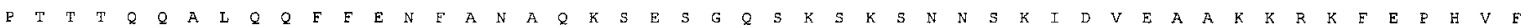
AATATARAGAGAGAGATGTAATGCTTTATGCGTTAGGATTGGCGCAACTCGCAAAGACTTGCAATGGGTITATGAAAACAGTGACAATTICTCTGTTATACCAA.CATTTGGGTCATCCCAGCAATTATTCTTTCCAATACTTTGCCAT

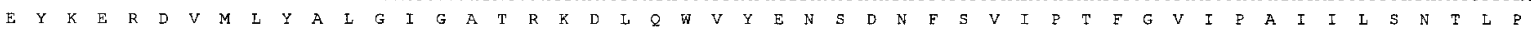

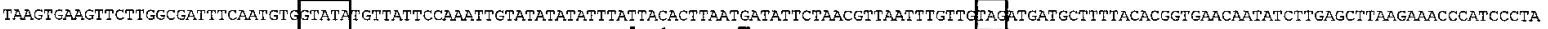

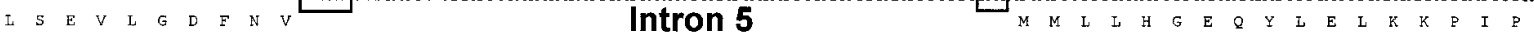
CTTCTGGAAATTAATTTCAACACCGTATGTTATTGATATTCTTGATAAAGGGAAGGGAGT GTCGTTTATTTTCGGTATAACTACTACAGATGAAAAAGGAGAAGTTATATTTGAAAATCAGACAACTCTATTTATTCGTGGTATTGGAC

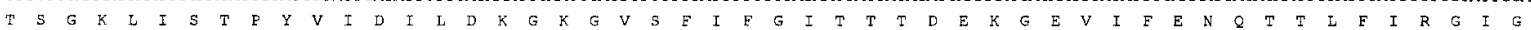
GTTTTGGCGGAAAAAAGACTGGTGATGATCGTGGTGCTGCTACAGCTTCAAACATTCCACCAAAACGCGCTCCAGATGTTGTAGTAAAGGAGAAAACAAATGAAAATCAAGCTGCTTTATATCGTTTAAGTGGAGATTATAATCCATTGC

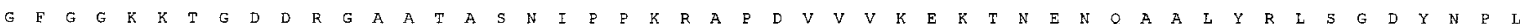
ATATTGATCCTAGTATGTCCGCAATGGGAGGTTTTGATGTTCCTATTCTTCATGGAATGTGTACCTTTGGTATATCCGGTAAACATATTTTTTCTACATTTGGTAAAAATGATCCAAATACTTTCAAGAGTATCAAAGCTCGATTAGCA.G

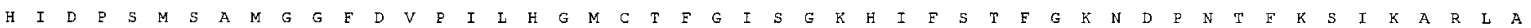

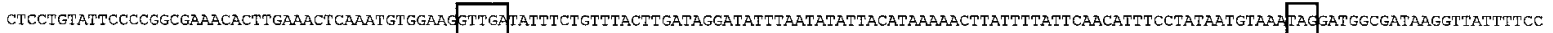
A $P$ P $V$ V F Intron 6 D G $\quad$ D $\quad K \quad V \quad I \quad F$ AAACCCETGTAGTTGAACGCGATGTCATCTETATTGCGTCTGCTGCTGTAGAGCTAAGAGGTTCTTCAGCTTCTGGGGCTAGTAT GGGAGCTCCCTCAGCAACTCCAAGTGCTGGTATTPCTGTACCTGGATTCCAATCATCTTCCGTAI \& $T$ T $R$ V V V E TTGAGCAGCTCAAAGCAGGTTTGGATGCTGCTTCTCCAGCGGAACGGCAAGCACAAGTAAAGAA GTATGGTGTGTTTTTATATATTIAATTAATTATT GAATCGCGAAAATTTAATTAATATTTTAATTACAATTTTATTTARTTTTTA F $\quad E \begin{array}{llllllllllllllllllllllllllll} & & L & K & A & G & L & D & A & A & S & F & A & E & R & Q & A & Q & V & K & K\end{array}$ Intron 7

AACTTMA AaGGTAAAAGGTTCTITCCAATTGAATATTAAAAAATGCTGAAGGCAAGGAACAATCATGGTACATAGACTTTAAGACGGGAGATGGTGCAGTTGGCATTGGACCATCACCAAAGAAAGCTGACGCAACCATT GGAGTTAGTGA

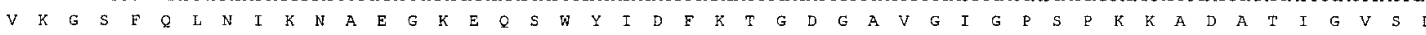

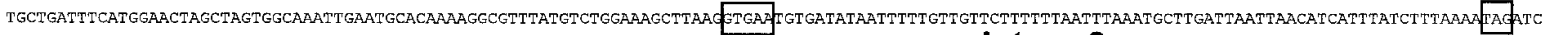
$\begin{array}{llllllllllllllllllllllllllllllllll}A & D & F & M & E & L & A & S & G & K & L & N & A & Q & K & A & F & M & S & G & K & L & K & & \end{array}$

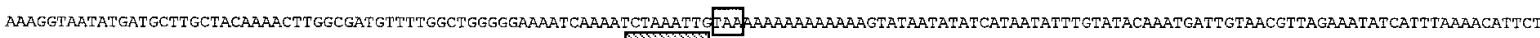

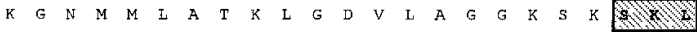

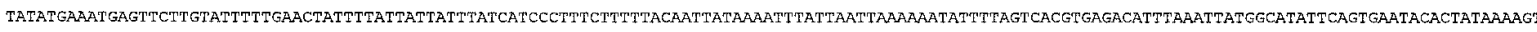
GGATATCATCATGT GGTITTACAACATCATAAATTATACATTCGTTCCTGCGTTTTCAGATIATTGCGCAGTTAAAAAA.CAAAATCATT GTTTTTAAAAAAGCCAAATATATCTCAAAAATAAAATATTTGAMATAAGCGTAT GAATTGT AAATTTICGTAAAGGAAAAAAAAATATATCAATTTTTATTTCTTTT

\section{0}

1800

Fig. 2. Gene structure of GmFOX2 gene from Glomus mosseae. The genomic sequence including positions of the introns and the deduced amino acid sequence are shown. The SKL motif of targeting to peroxisomes is highlighted. The exon/intron boundaries are indicated. The GmFOX 2 sequence data have been deposited in the EMBL data base as accession number AJ243538. 
cose repression and oleate induction are responsible for the transcriptional regulation of all the peroxisomal genes from yeast, which share common sequence regulatory elements in the promoter region (Einerhand et al. 1995). Therefore, it is likely that the repression of GmFOX2 could be established as a consequence of a glucose repression stage. Glucose repression is a common cell mechanism in fungi to sense the presence of easily available or preferred carbon sources such as glucose and in consequence decrease the expression of enzymes required for the use of other more complex carbon sources such as lipids (Ebbole 1998; Gancedo 1998; Johnston 1999). In AM fungi there is evidence that during the first 5 days after germination trehalose is preferentially used over triacylglycerides despite the low abundance of this sugar in AM spores (Bécard et al. 1991; Bécard 1998). Trehalose breakdown provides the cell with glucose that feeds into the glycolysis pathway. In other fungi, including some zygomycetes, trehalose is mobilized during germination with the participation of neutral trehalases activated upon phosphorylation by cyclic AMP (cAMP)-dependent protein

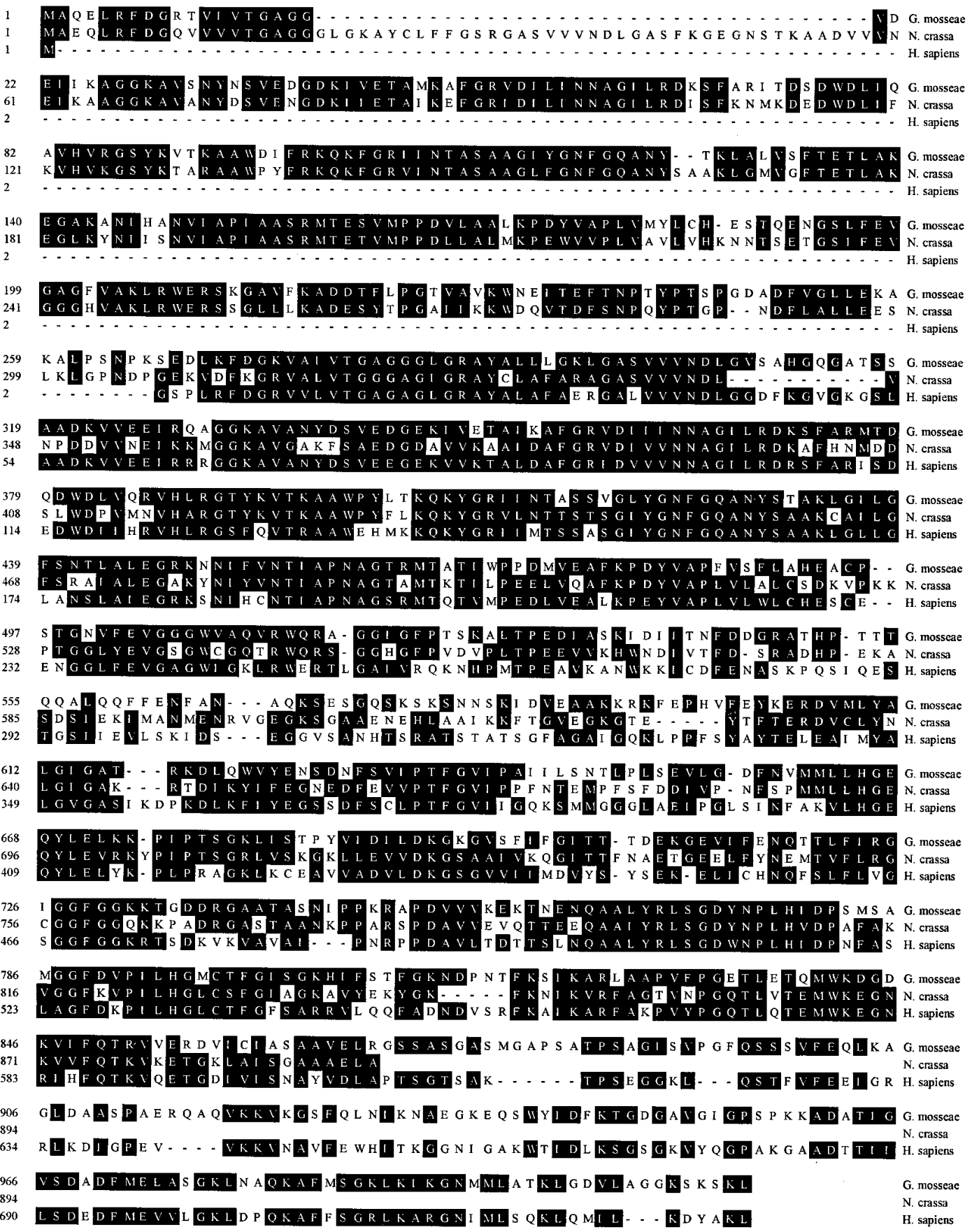

Fig. 3. Alignment of the deduced amino acid sequence of GmFOX2 with amino acid sequences of the Neurospora crassa FOX2 (S54786) and the human 17ß-HDS IV (p51657). Bold areas represent conserved amino acids. 
kinase (PKA) (Van Laere 1995; Thevelein 1996; d'Enfert et al. 1999).

The question is, of course, how the bacteria are signaling the fungus to produce such a glucose repression stage. A clue for that comes from the dual role of the first domain of the human homologue of GmFOX2 and from the nod $G$ gene from $R$. meliloti. In humans, the down-regulation of 17-HDS IV increases the level of active estrogens that could then mediate their mitogenic role (Carstensen et al. 1996). In $R$. meliloti, nod $\mathrm{G}$ is one of the genes responsible for the nodulation regulated upon the onset of flavonoid signals exuded from the plant. Recently, it has been found that certain specific flavonoids such as coumestrol are able to exert a repressor activity on nod gene expression (Zuanazzi et al. 1998). Flavonoids and estrogens are structurally similar molecules. In AM fungi the exogenous addition of $17 \beta$ estradiol was found to increase the asymbiotic growth of $G$. intraradices (Poulin et al. 1997) because of the structural similarities between estradiol and biochanin A, a plant isoflavone that is known to stimulate mycorrhizal presymbiotic growth. Estrogens are present in fungal cells as minor components, but their role is not clear. Interestingly, it was shown that in yeast the exogenous addition of $17 \beta$ estradiol was able to provoke the recovery of growth from cells arrested in G0 (as a consequence of nutrient starvation or mutation of the adenylate cyclase gene) by increasing the intracellular level of cAMP (Tanaka et al. 1989). In mammalian cell cultures, specific flavonoids have been reported as inactivators of the cAMP phosphodiesterase, thereby raising the cAMP level in the cell. High cAMP levels are thought to mediate glucose repression, although the mechanism by which this happens remains obscure (for review see Gancedo 1998). Therefore, in G. mosseae the production of a flavonoid/estrogen signal from the bacteria could mediate an increase in cAMP responsible for the glucose repression stage that down-regulates GmFOX2. Similarly, the gene FOX3 from yeast, encoding the peroxisomal thiolase, requires respiration (de-repressed stage) and low cAMP-

Table 3. Densitometric analysis of the quantitative reverse transcriptionpolymerase chain reaction shown in Figure $5^{\mathrm{a}}$

\begin{tabular}{|c|c|c|c|c|c|c|}
\hline \multirow{2}{*}{$\begin{array}{l}\text { Polymerase } \\
\text { chain reaction } \\
\text { product }\end{array}$} & \multicolumn{3}{|c|}{$24 \mathrm{~h}$} & \multicolumn{3}{|c|}{$120 \mathrm{~h}$} \\
\hline & $\mathrm{C}$ & B & $\mathbf{M}$ & $\mathrm{C}$ & B & $\mathbf{M}$ \\
\hline GmFOX2 & 19 & 9 & 13 & 15 & 13 & 9 \\
\hline Mimic & 15 & 16 & 16 & 18 & 16 & 16 \\
\hline
\end{tabular}

a Standard deviation for all data is $<0.009$.

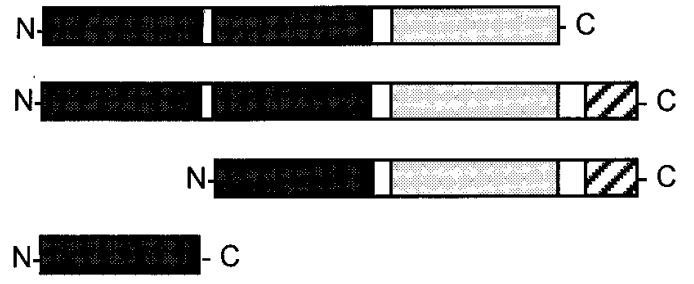

FOX2 N. crassa

GmFOX2 G. mosseae

17ß-HDS H. sapiens

NodG R. meliloti

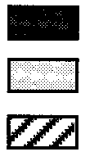

dehydrogenase domain

hydratase domain

sterol carrier-protein domain

Fig. 4. Domain distribution in GmFOX2 and its homologues from Neurospora crassa, human, and Rhizobium meliloti genes.

\section{$24 \mathrm{~h}$}

$120 \mathrm{~h}$

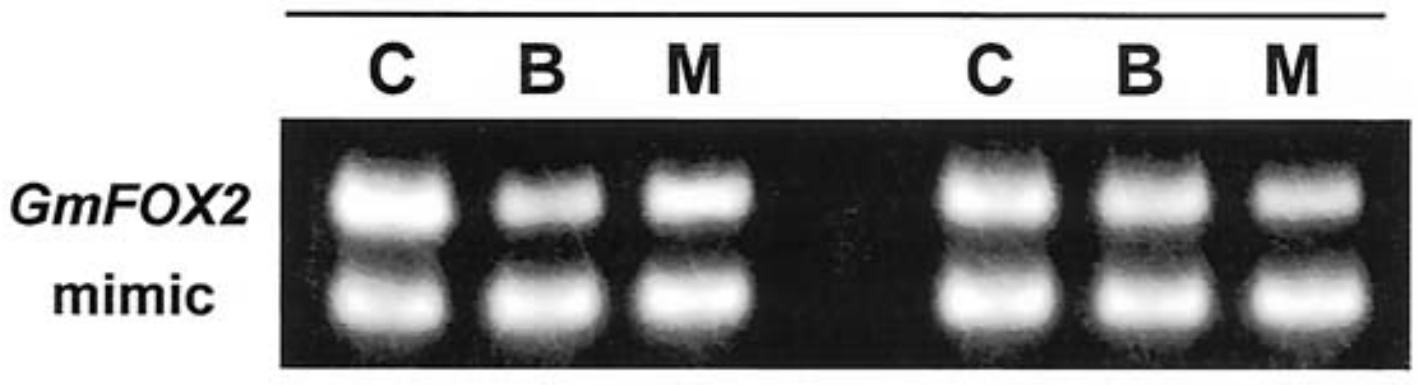

rRNA
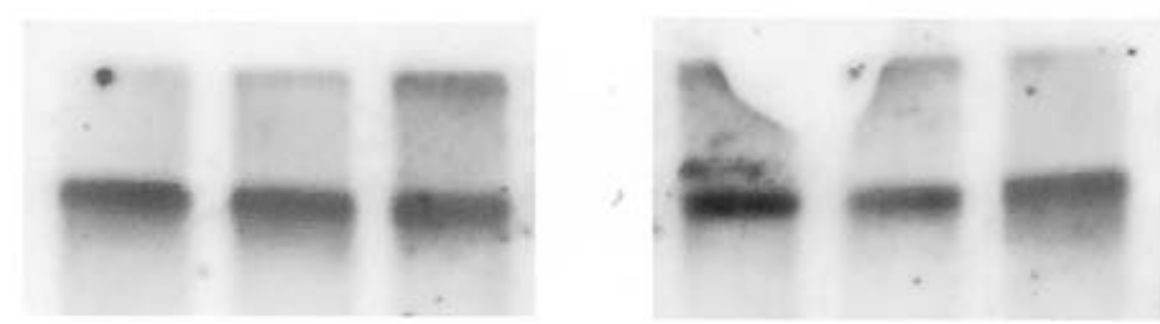

Fig. 5. Competitive reverse transcription-polymerase chain reaction (RT-PCR) analysis of the expression of GmFOX2 in Glomus mosseae at two different time points of growth, 24 and $120 \mathrm{~h}$, in sporocarps inoculated either with saline solution (C), Bacillus subtilis NR1 (B), or Mesorhizobium mediterraneum NR2 (M). Standard amounts of RNA for each sample were calibrated by Northern (RNA) blot with the ribosomal RNA genes of $G$. mosseae as a probe. The mimic concentration used in this experiment was 5 amol. 
dependent PKA activity to be expressed at a high level (Igual and Navarro 1996). It will be the challenge of future research to unravel the role of cAMP in the regulation of GmFOX2 and the links to fungal growth during presymbiotic stages.

\section{ACKNOWLEDGMENTS}

We thank R. Fischer and G. Bécard for their fruitful scientific discussions. This work was supported by the European Commission with a Marie-Curie Postdoctoral Research Training Grant to N. R.

\section{LITERATURE CITED}

Azcón, R. 1989. Selective interaction between free-living rhizosphere bacteria and vesicular-arbuscular mycorrhizal fungi. Soil Biol. Biochem. 21:639-644.

Azcón-Aguilar, C., and Barea, J. M. 1992. Interactions between mycorrhizal fungi and other rhizosphere microorganisms. Pages 163-198 in: Mycorrhizal Functioning: An Integrative Plant-Fungal Process. M. J. Allen, ed. Chapman and Hall, New York.

Azcón-Aguilar, C., Diaz-Rodriguez, R. M., and Barea, J. M. 1986. Effect of soil micro-organisms on spore germination and growth of the vesicular-arbuscular mycorrhizal fungus Glomus mosseae. Trans. Br. Mycol. Soc. 86:337-340.

Barea, J. M., and Jeffries, P. J. 1995. Arbuscular mycorrhizas in sustainable soil-plant systems. Pages 521-560 in: Mycorrhiza. A. Varma and B. Hock, eds. Springer-Verlag, Berlin.

Bécard, G. 1998. Mechanisms controlling growth and development of arbuscular mycorrhizal fungi. In: Abstracts from the Sixth International Mycological Congress, Jerusalem.

Bécard, G., Doner, L. W., Rolin, D. B., Douds, D. D., and Pfeffer, P. E. 1991. Identification and quantification of trehalose in vesiculararbuscular mycorrhizal fungi by in vivo ${ }^{13} \mathrm{C}$ NMR and HPLC analyses. New Phytol. 118:547-552.

Berbee, M. L., and Taylor, J. W. 1992. Dating the evolutionary radiations of the true fungi. Can. J. Bot. 71:1114-1127.

Bonfante, P., and Bianciotto, V. 1995. Presymbiotic versus symbiotic phase in arbuscular endomycorrhizal fungi: Morphology and cytology. Pages 229-247 in: Mycorrhiza. A. Varma and B. Hock, eds. Springer-Verlag, Berlin.

Budi, S. W., Blal, B., and Gianinazzi, S. Surface-sterilization of Glomus mosseae sporocarps for studying endomycorrhization in vitro. Mycorrhiza (In press.)

Calvet, C., Barea, J. M., and Pera, J. 1992. In vitro interactions between the vesicular-arbuscular mycorrhizal fungus Glomus mosseae and some saprophytic fungi isolated from organic substrates. Soil Biol. Biochem. 24:775-780.

Carpenter-Boggs, L., Loynachan, T. E., and Stahl, P. D. 1995. Spore germination of Gigaspora margarita stimulated by volatiles of soilisolated actinomycetes. Soil Biol. Biochem. 27:1445-1451.

Carstensen, J. F., Tesdorpf, J. G., Kaufmann, M., Markus, M. M., Husen, B., Leenders, F., Jakob, F., de Launoit, Y., and Adamski, J. 1996. Characterization of the $17 \beta$-hydroxysteroid dehydrogenase IV. J. Endocrinol. 150:3-12.

d'Enfert, C., Bonini, B. M., Zapell, P. D. A., Fontaine, T, da Silva, A. M., and Terenzi, H. F. 1999. Neutral trehalases catalyse intracellular trehalose breakdown in the filamentous fungi Aspergillus nidulans and Neurospora crassa. Mol. Microbiol. 32:471-483.

Ebbole, D. J. 1998. Carbon catabolite repression of gene expression and conidiation in Neurospora crassa. Fungal Genet. Biol. 25:15-21.

Einerhand, A. W., Kos, W., Smart, W. C., Kal, A. J., Tabak, H. F., and Cooper, T. G. 1995. The upstream region of FOX3 gene encoding peroxisomal 3-oxoacyl-coenzyme A Thiolase in Saccharomyces cerevisiae contains ABF1- and replication protein A-binding sites that participate in its regulation by glucose repression. Mol. Cell. Biol. 15: 3405-3414.

Fossa, A., Beyer, A., Pfitzner, E., Wenzel, B., and Kunau, W. H. 1995. Molecular cloning, sequencing and sequence analysis of the fox-2 gene of Neurospora crassa encoding the multifunctional $\beta$-oxidation protein. Mol. Gen. Genet. 247:95-104.
Franken, P., and Gianinazzi-Pearson, V. 1996. Construction of genomic libraries of the arbuscular mycorrhizal fungi Glomus mosseae and Scutellospora castanea and isolation of ribosomal RNA genes. Mycorrhiza 6:167-173.

Gancedo, J. M. 1998. Yeast carbon catabolite repression. Microbiol. Mol. Biol. Rev. 62:334-361.

Gaspar, M. L., Pollero, R., and Cabello, M. 1994. Triacylglycerol consumption during spore germination of vesicular-arbuscular mycorrhizal fungi. J. Am. Oil Chem. Soc. 71:449-452.

Gurr, S. J., Unkles, S. E., and Kinghorn, J. R. 1987. The structure and organization of nuclear genes of filamentous fungi. Pages 94-139 in: Gene Structure in Eukaryotic Microbes. J. R. Kinhorn, ed. IRL Press, Oxford.

Hepper, C. 1979. Germination and growth of Glomus caledonius spores: The effects of inhibitors and nutrients. Soil Biol. Biochem. 2:269-277.

Hosny, M., van Tuinen, D., Jacquin, F., Füller, P., Zhao, B., GianinazziPearson, V., and Franken, P. 1999. Arbuscular mycorrhizal fungi and bacteria: How to construct prokaryotic DNA-free genomic libraries from the Glomales. FEMS Microbiol. Lett. 170:425-430.

Igual, J. C., and Navarro, B. 1996. Respiration and low cAMPdependent protein kinase activity are required for high-level expression of the peroxisomal thiolase gene in Saccharomyces cerevisiae. Mol. Gen. Genet. 252:446-455.

Johnston, M. 1999. Feasting, fasting and fermenting. Trends Genet. $15: 29-33$

Kunau, W. H., and Hartig, A. 1992. Peroxisome biogenesis in Saccharomyces cerevisiae. Antonie van Leeuwenhoek 62:63-78.

Leenders, F., Tesdorpf, J. G., Markus, M., Engel, T., Seedorf, U., and Adamski, J. 1996. Porcine 80-Kda protein reveals intrinsic 17 $\beta$ hydroxysteroid dehydrogenase, fatty acyl CoA-hydratase/dehydrogenase and sterol transfer activities. J. Biol. Chem. 271:5438-5442.

Martin-Laurent, F., van Tuinen, D., Dumas-Gaudot, E., GianinazziPearson, V., Gianinazzi, S., and Franken, P. 1997. Differential display analysis of RNA accumulation in arbuscular mycorrhiza of pea and isolation of a novel symbiosis-regulated plant gene. Mol. Gen. Genet. 256:37-44.

Mayo, K., Davis, R. E., and Motta, J. 1986. Stimulation of germination of spores of Glomus versiforme by spore-associated bacteria. Mycologia 78:426-431.

Mosse, B. 1959. The regular germination of resting spores and some observations on the growth requirements of an Endogone sp. causing vesicular-arbuscular mycorrhiza. Trans. Br. Mycol. Soc. 42:273-286.

Mosse, B. 1988. Some studies relating to 'independent' growth of vesicular-arbuscular endophytes. Can. J. Bot. 66:2533-2540.

Mugnier, J., and Mosse, B. 1987. Spore germination and viability of a vesicular arbuscular mycorrhizal fungus, Glomus mosseae. Trans. Br. Mycol. Soc. 88:411-413.

Persson, B., Krook, M., and Jörnvall, H. 1991. Characteristics of shortchain alcohol dehydrogenases and related enzymes. Eur. J. Biochem. 200:537-543.

Poulin, M.-J., Simard, J., Catford, J.-G., Labrie, F., and Piché, Y. 1997. Response of symbiotic endomycorrhizal fungi to estrogens and antiestrogens. Mol. Plant-Microbe Interact. 10:481-487.

Requena, N. 1998. Mycorrhizal interactions in the rhizosphere. Pages 725-738 in: Microbes for Health, Wealth and Sustainable Environment. A. Varma, ed. Malhorta Pub. House, New Delhi.

Requena, N., Jeffries, P. J., and Barea, J. M. 1996. Assessment of natural mycorrhizal potential in a desertified semiarid ecosystem. Appl. Environ. Microbiol. 62:842-847.

Requena, N., Jimenez, I., Toro, M., and Barea, J. M. 1997. Interactions between plant-growth-promoting rhizobacteria (PGPR), arbuscular mycorrhizal fungi and Rhizobium spp. in the rhizosphere of Anthyllis cytisoides, a model legume for revegetation in Mediterranean semiarid ecosystems. New Phytol. 136:667-677.

Seedorf, U., Raabe, M., Ellinghaus, P., Kannenberg, F., Fobker, M., Engel, T., Denis, S., Wouters, F., Wirtz, K. W. A., Wanders, R. J. A., Maeda, N., and Assmann. G. 1998. Defective peroxisomal catabolism of branched fatty acyl coenzyme A in mice lacking the sterol carrier protein-2/sterol carrier protein-x gene function. Genes Dev. 12:11891201.

Sheldon, P. S., Kekwick, R. G. O., Sidebottom, C., Smith, C. G., and Slabas, A. R. 1990. 3-Oxoacyl-(acyl-carrier protein) reductase from avocado (Persea americana) fruit mesocarp. Biochem. J. 271:713-720.

Simon, L., Bousquet, J., Lévesque, R. C., and Lalonde, M. 1993. Origin 
and diversification of endomycorrhizal fungi and coincidence with vascular plants. Nature 363:67-69.

Tanaka, S., Hasegawa, S., Hishinuma, F., and Kurata, S. 1989. Estrogen can regulate the cell cycle in the early G1 phase of yeast by increasing the amount of adenylate cyclase mRNA. Cell 57:675-681.

Thevelein, J. M. 1996. Regulation of trehalose metabolism and its relevance to cell growth and function. Pages 395-420 in: Mycota III. Biochemistry and Molecular Biology. R. Brambl and G. A. Maryluf, eds. Springer-Verlag, Berlin.

Tylka, G. L., Hussey, R. S., and Roncadori, R. W. 1991. Axenic germination of vesicular-arbuscular mycorrhizal fungi: Effects of selected
Streptomyces species. Phytopathology 81:754-759.

Van Laere, K. 1995. Intermediate metabolism. Pages 211-238 in: The Growing Fungus. N. A. R. Gow and G. M. Gadd, eds. Chapman and Hall, London.

Wilson, G. W. T., Hetrick, B. A. D., and Kitt, D. G. 1989. Suppression of vesicular-arbuscular mycorrhizal fungus spore germination by nonsterile soil. Can. J. Bot. 67:18-23.

Zuanazzi, J. A. S., Clergeot, P. H., Quirion, J.-C., Husson, H.-P., Kondorosi, A., and Ratet, P. 1998. Production of Sinorhizobium meliloti nod gene activator and repressor flavonoids from Medicago sativa roots. Mol. Plant-Microbe Interact. 11:784-794. 